\title{
Comparison of Two Numerical Techniques for Aerodynamic Model Identification
}

\section{H. Verhaegen}

\author{
(AAS:-TH-3946/) CCAEAFISCA CF THC NUEEEICAD \\ N87-249:9 \\ ILCE: ICU'S ECT AEFOCYNAUIC ECLEL \\ ILENTIFICATIC (BASA) 11 F AVail: MIIS \\ LC $102 / \mathbb{B} \triangle 01$ \\ $\operatorname{CSCL} 12 \mathrm{~A}$ \\ $\begin{array}{ll}\text { Unclas } \\ 63 / 65 & 0082710\end{array}$
}

June 1987 


\section{Comparison of Two Numerical Techniques for Aerodynamic Model Identification}

M. H. Verhaegen, Ames Research Center, Moffett Field, California

June 1987

\section{N/SA}

National Aeronautics and

Space Administration

Ames Research Center

Moffett Field, California 94035 
COMPARISON OF THO NUMERICAL TECHNIQUES FOR AERODYNAMIC MODEL IDENTIFICATION

M. H. Verhaegen"

NASA Ames Research Center, Moffett Fleld, California

\section{Abstract}

A new algorithn, called the Minimal Residual QR algorithm, is presented to solve subset regression problems. It is shown that this new scheme can be used as a numerically rellable implementation of the stepwise regression technique, which is widely used to Identify an aerodynamic model from flight test data. This capability as well as the numerical superiority of this scheme over the stepwise regression technique is demonstrated in an experimental simulation study.

\section{Introduction}

This paper describes a study of the so-called subset regression problem (SRP). This problem occurs in the Identification of a mathematical model representing the aerodynamic forces and moments which act on an aircraft as a function of i) aircraft state quantities, such as angie of attack and Mach number and 2) alreraft input quantitles, such as control surface deflections. This mathematical model is referred to as the aircraft's aerodynamic model. In Refs. 1-3, precise conditions have been stated which allow formulation of this Identification problem as an SRP.

In this paper, we focus on the numerical technlques used to solve SRP. Generally, techniques for solving SRP are divided into two different classes:

1) A first class is formulated in a complete statistical framework. The techniques in this class are generally referred to as subset regression methods and are widely used by practicing engineers and econometrists. 4

2) A second class has its basis mainly in numerlcal analysis. Currently the most widely used technique from this class is the singular Value Decomposition (SVD) method. 5

The SVD has clearly demonstrated its numer1cal superiority over the techniques from the first class. However, It does not do the subset selection from the originally defined model parameters. This is a major drawback for the aerodynamle model identification problem, since the original model parameters have a physical interpretation.

In order to overcome this drawback, a new algorithm has been developed. This new technique

\section{Assoclate of the U.S. National Research} Councll.

This paper is declared a work of the U.S. Government and therefore is in the public domain.
Is called the Minimal Residual QR (MRQR) algo$r$ Ithm. Although the MRQR scheme is derived from a numerical analysis point of view and therefore belongs in the second class mentioned above, it will be shown that it combines the advantages of techniques from both classes. On the one hand, it retalns the precision of the original data such as the SVD and on the other hand can be used In a complete similar way as the stepwise regression technique (SRT), since it produces quantities such as sequential F-tests, etc.

The outline of the paper is as follows. In Section 2, the new scheme will be described. Its relationship with the existing SRT is indicated in Section 3, and Section 4 presents the result of a comparison study between the MROR and the SRT. Finally, Section 5 presents the conclusions of this research.

\section{The iinimai Kesiduai Of Algor i inm}

This new scheme is originally proposed in Ref. 7. In this section, we review this scheme to reveal the relationship with the SRT.

The MRQR algorithm performs a QR factorization with column pivoting of the system matrix $A$ in the considered SRP. If this SRP is denoted as :

$$
\operatorname{mintax}_{x}-b l_{2}
$$

with $I_{2} I_{2}$ representing the Euclidean norm, and

$$
A \in R^{m \times n}(m \geq n), \quad b \in R^{m}, \quad x \in R^{n}
$$

and

$$
\operatorname{rank}(A)=k \leq n
$$

then we can write the result of MRQR as

$$
\min _{x} Q^{T} A \pi\left(\pi^{T} x\right)-Q^{T} b_{2}
$$

where 9 is an orthogonal transformation matrix, 1.e., $Q_{Q}=I$ and $\pi$ is the column permutation matrix. The operation Indicated by Eq. (2) results in

$$
\underset{x}{\min }\left\{\left[\begin{array}{c|c}
\mathrm{R}_{11} & \mathrm{R}_{12} \\
\hline 0 & \mathrm{R}_{22}
\end{array}\right]\left[\frac{\mathrm{x}_{1}}{\mathrm{x}_{2}}\right]-\left.\left[\frac{\mathrm{b}_{1}}{\mathrm{c}}\right]\right|_{2}\right.
$$

where $R_{11}$ is $k \times k$ and upper triangular, $R_{12}$ is $k \times(n-k)$ and $b_{1} \in R^{k}$. From the decomposition of $\mathrm{Eq}$. (3), the solution to Eq. (1), which now is no longer a minimal norm solution, becomes 


$$
\hat{x}_{1}=R_{11}^{-1} b_{1}
$$

and the 2-norm of the residual is given by $\mid \in \|_{2}$.

The cruclal part in this computational scheme [Eqs. (2-4)] is the generation of the column permutation matrix $\pi$. The way this is done In the MRQR scheme is now lllustrated for the simple twodimensional case.

\subsection{The Two-Dimensional Case}

In this case, the data of problem (1), l.e., the two-column vectors of $A$ and the right hand side (rhs) b, are graphlcally represented in Fig. 1 .

Just as with the SRT, the MRQR algorithm selects one column vector of $A$ at a time. The measure Initlally proposed in Ref. 7 for this selection is "the distance of the rhs to the column vectors of A."

In Fig. 1, these distances are denoted by $m_{l}$, where the superscript Indicates that we are selecting the first column and the subscript Ind Icates the corresponding column position in the A-matrix. The column that will be selected by the MRQR algorithm is the one "closest" to the rhs. This corresponds to finding the minimum of the sequence $(1 \mathrm{~m}, \mathrm{l})$, which for the case of $\mathrm{Fig} .1$ results in the selection of $a_{1}$. This column is then permuted to the first column position of A. In the next step, the same selection procedure is repeated for the components of the remaining columns of $A$ and the component of the rhs that is orthogonal to $a_{1}$. These components are represented in Fig. 1 by $a_{2}^{2}$ and $b^{2}$, respectively. The selection in the orthogonal complement of $a_{1}$ is obvious now, since $\left|m_{2}^{2}\right|=0$.

The quantities $\mid m^{1}$ | will be referred to as "residuals" because each $\mid m_{l}^{\prime}$ is the residual of the least squares problem

$$
\operatorname{minla}_{e}^{1} \xi-b^{1} 1_{2}
$$

(Ref. 7). The selection of the minimum of that sequence (ImI) gave rise to the algorithmic name, Minimal Residual QR algorithm.

\subsection{Generallzat Ion of the Two-Dimensional Case}

The generalization of the two-dimensional case, given in pseudo-programing-language form, is summarized in the following algorithm.

\section{Algorithm 1:}

Define:

$$
\begin{aligned}
& A=A^{1}=\left[a_{1}^{1} \ldots a_{j}^{1} \ldots a_{n}^{1}\right] \text { and } b^{1}=b \\
& \text { rank }=n
\end{aligned}
$$

DO

$$
1=1: n
$$

1) Select column vector of $A^{i}$ closest to $b^{i}$, based on the computed sequence of residuals (imil). This column is called $a_{j}$.

2) Interchange the $\mathrm{jth}$ and ith column vector of $A^{1}$ by the column permutation matrix $\pi_{1}$.

3) Perform an orthogonal projection $Q_{i}$, such that

$$
\begin{aligned}
& Q_{1}^{T}\left[\begin{array}{c|cc}
R_{11}^{(1-1)} & R_{12}^{(1-1) \times(n-1+1)} \\
\hline 0 & a_{1}^{i} \ldots a_{j}^{i} \ldots a_{n}^{i}
\end{array}\right] \pi_{1}
\end{aligned}
$$

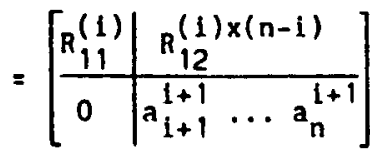

$$
\begin{aligned}
& =\left[\begin{array}{c|c}
R_{11}^{(i)} & R_{12}^{(1) \times(n-i)} \\
\hline 0 & A^{i+1}
\end{array}\right]
\end{aligned}
$$

and

$$
Q_{i} b^{i}=\left[\frac{w}{b^{i+1}}\right]^{b i}
$$

where $R_{11}^{(1)}$ is an ixi upper-triangular matrix and $R_{12}^{(1) \times(j)}$ is an $1 \times \mathrm{J}$ rectangular matrix.

4) Rank determination test.

\section{END}

The rank determination in the fourth step of the in-loop, 1.e., rejecting one of the columns of $A$, can be done in various ways. In this paper, this determination will be based on the so-called partial F-test quantities, such as those used in SRT. ${ }^{4}$ This is outlined in section 3 .

\subsection{Implementation Note on the MRQR Algor Ithm}

An efficient way to calculate the residuals I $m_{l} \mid$ and to perform the orthogonal transformations $Q_{1}$ in Eq. (6) is described in Ref. 7 . Here we initially transform problem (1) to:

$$
\underset{x}{\min \| Q_{h}^{T} A x}-Q_{h}^{T_{b} l_{2}}=\underset{x}{\min }\left|\left[\frac{R}{0}\right] x-\left[\frac{b^{\prime}}{0}\right]\right|_{2}
$$

where $R$ is an $n \times n$ upper-triangular matrix and $b^{1} \in R^{n+1}$.

This compression of the data can be done sequentlally, such as with the construction of the "normal" equations (see section 3 ). This sequential ability also makes this scheme attractive from the data storage point of vlew. 
Next, Algorithm 1 is applied to the righthand side of Eq. (7). A combination of these intermediate steps would then result in the following reformulation of problem (1).

$$
\begin{aligned}
& \min _{x} \|\left[Q_{k}^{T} \ldots Q_{1}^{T}\right] Q_{h}^{T} A\left[\pi_{1}, \ldots \pi_{k}\right]\left(\left[\pi_{1} \ldots \pi_{k}\right]^{T} x\right) \\
& -10_{k}^{T} \ldots Q_{1}^{T} 10_{h}^{T} b_{2}
\end{aligned}
$$

where $\left[Q_{k}^{T} \ldots Q_{1}^{T}\right]$ will be denoted as $Q_{g}$ and

Note: The robustness properties of the MRQR algorithm rely on the use of orthogonal transformations, denoted in $E q .(8)$ by $Q_{h}, Q_{g}$, and $\pi$, respectively. These do not modify the error pattern present on the original data. 6

\section{Relationship of the MRQR Algor Ithm with Stepwise Regression}

The classical stepwise regression technique (SRT) solves problem (1) via the so-called normal equations.

$$
\left(A^{T} A\right] x=A^{T} b
$$

From this set of equations the partial F-test quantities, the partial correlation coefficients, and other quantities are computed. ${ }^{4,8}$ These latter quantities form the crucial information source in adding (or subtracting) a new variable $x_{1}$ in the regression model. The partial F-test quantitles are defined next.

Definition 1. When in the construction of the regression model, we add parameter $x_{l}$ to the model already containing $i-1$ parameters, the F-test value for that parameter $x_{l}$ is:

$$
F_{x_{\ell}}=\frac{\varepsilon_{1-1}^{T} \varepsilon_{1-1}-\varepsilon_{1}^{T} \varepsilon_{1}}{\varepsilon_{1}^{T} \varepsilon_{1}}(m-1)
$$

where $\varepsilon_{1-1}$, $\varepsilon_{1}$ are the restduals of regression models containing $1-1$ and 1 parameters, respectively.

The quantity defined in Eq. (10) can also be derived from the MRQR algorithm. In order to clarify this, let us focus at the ith stage of the do-loop of algorithm 1. The partlal F-test value for each parameter $x_{l}$ not yet in the model becomes:

$$
F_{x_{L}}=\frac{\left\|b^{1}\right\|_{2}^{2}-\left|m_{l}^{1}\right|_{2}^{2}}{\left|m_{l}^{1}\right|_{2}^{2}}(m-1)
$$

The difference of the two norms in Eq. (II) does not have to be computed explicitly; it becomes available during the computation of Im $I_{2}$. If we denote this difference by Id $\left.\right|_{2}$, then $\mathrm{Eq}$. (11) becomes

$$
F_{x_{l}}=\frac{\operatorname{Id}_{l}^{1} \|_{2}^{2}}{\operatorname{lm}_{l} \|_{2}^{2}}(m-1)
$$

The F-test quantitles now allow us to make a decision about the statistical significance of adding parameter $x_{2}$ to the model. Statistical significance corresponds to the $F$-test values being above a threshold $F_{a}$, which is taken from statistical tables about the F-distribution (e.B., Hef. 4). Erom Eq. (12), we clearly see that we can impose this test directly on the elements of the derived residual sequence $\left(1 \mathrm{~m}_{l}{ }^{\prime}{ }_{2}\right)$ in the MRQR algorithm. Based on the desired threshold $F_{a}$, such a test would become

$$
\left\|m_{l}^{l}\right\|_{2} \leq \frac{\left|d_{l}^{1}\right|_{2} \sqrt{(m-i)}}{\sqrt{F_{a}}}
$$

This inequality precisely reveals the relationship between the MRQR and SRT. It indicates that the same parameter $x_{2}$ would be added to the model whether based on the $\left(\operatorname{Im}_{2} 1_{2}\right)$ sequence or the [ $\mathrm{F}_{\mathrm{x}_{\ell}}$ ] sequence.

This section summarizes the use of the MRQR algorlthm as a robust implementation of the classical stepwise regression scheme. Furthermore, as demonstrated in Ref. 7, the MRQR algorithm gives rise to additional parameters, such as smallest singular values, that may be helpful in constructing the regression model. In this paper, such an additional feature is presented in section 4.2.

\section{Experimental Evaluation}

\subsection{Demonstration of the Equivalence between MRQR and SRT}

In this first example, the data taken from Ref. 9 (p. 647) and analyzed In Ref. 4 via the classical SRT are analyzed by the proposed MRQR algorithm. These data, summarized in Table 1, comprise four candidate solutions (i.e., the columns of the $A$ matrix), and a right-hand side (the b-vector), from which 13 samples have been recorded. Hence, $m=13$ and $n=4$. In this section the candidate solutions will be referred to by their corresponding component of the $x$-vector, defined in $\mathrm{Eq}$. (1). The results of the MRQR algorithm are summarlzed in Table 2 . ComparIson of the partial F-test values derived from MRQR, denoted by $F_{1}$, with those derlved from SRT, denoted by $F_{2}$, clearly demonstrates the equivalence of both schemes. Furthermore, we also observe that using the $\left(1 \mathrm{~m}_{2} \mathrm{l}_{2}\right)$ sequence results in the same model as using the partial F-test values.

\subsection{An Additional Feature of the MRQR}

In analyzing real data, visual inspection of time-history plots of the avallable data is often 
used. Such plots of the candidate solutions and the rhs mlght allow (or influence) the decision of which parameter to include in the regression model or might help to judge the validity of the extracted model. If the same graphical information for all subsequent decision stages is desired, it is generally necessary to store the original A-matrix and explicitly compute the residuals of the columns not yet in the model.

In-the ith decision stage, the matrix A is partitioned as

$$
A=\left[A^{l-1} \mid A^{n-l+1}\right]
$$

where $A^{1-1}$ designates the already-selected columns, then the residuals of the columns in $A^{n-i+1}$ become $\left[A^{i-T_{x}} x_{1-1}-A^{n-1+1}\right]$. These residuals can also be computed from the data avallable from the MRQR algorithm without expllcitly storing the A-matrix. Using Eqs. (6-8) they become

$$
Q_{h}\left[Q_{1} \ldots Q_{i-1}\right]\left|\begin{array}{lllll}
\frac{0}{1} & \ldots & 0 & \ldots & 0 \\
a_{1} & \ldots & a_{j} & \ldots & a_{n}^{i}
\end{array}\right|
$$

Here only $\left[Q_{1} \ldots Q_{1-1}\right]$ is stored explicitly, whereas the information to construct $\theta_{h}$ can be stored in the lower triangular part of $(R \mid 0]^{\top}$ in $\mathrm{Eq}$. (7). ${ }^{10}$ In this way, use of $\mathrm{Eq}$. (15) becomes a very rellable and storage-efficient way to evaluate residuals in the regression analysis.

This feature is demonstrated using the following example taken from Ref. 11 (p. ID-4.7). Here the original plant was generated by the following static model.

$$
\begin{aligned}
y(t) & =1.2 \sin (t)+2 \sin (.7 t)+3 \cos (t) \\
& -0.1[\sin (2 t)+8]-2 \sin (.99 t)+e
\end{aligned}
$$

where $\delta$ and $e$ are zero-mean white noise sequences with standard deviations of 0.001 and 0.1 , respectively.

In the regression analysis, the following model was postulated.

$$
\begin{aligned}
b(t)=x_{1} \sin (t)+x_{2} \sin (.7 t) & +x_{3} \cos (t) \\
& +x_{4} \cos (3 t)
\end{aligned}
$$

The four candidate solutions $[\sin (t)$, $\sin (.7 t), \cos (t), \cos (3 t))$ and $y(t)$ of Eq. (16) are shown in Fig. 2. Clearly, from this flgure candidate solution $x_{3}$ appears most closely related to the rhs, as was also demonstrated by the $F$-test values or the $\left.\left(1 m_{l}^{1}\right)_{2}\right)$ residuals. After this selection, the remaining parts of the other candidate solutions as well as the remaining part of the rhs are shown in Fig. 3 .
Next the candidate solution $x_{2}$ was selected. Agaln, Fig. 4 displays the residuals of the remaining columns and the rhs. From this figure the "high" correlation with $x_{1}$ becomes clear. The residuals of the remaining signals, l.e., rhs and $x_{4}$, are displayed in Fig. 4 .

From this analysis, we clearly observe that the residuals of each of the candidate solutions after each selection are nearly the same as the candidate solutions before the selections. This is a clear indication of "orthogonality" of the candidate solutions, which is also attributed to the value of the condition number of the matrix contalning the original four candidate solutions. The latter is $\approx 2$, which clearly is close to 1 .

This example demonstrates one feature of this "residual" analysis, but practical experience might provide additional uses.

\subsection{Numerical Super Iority of the MRQR}

In the previous two examples, the condition number of the original A-matrix, as defined in Eq. (1), was very close to 1. For these cases, the F-tests computed from the MRON algorithm or the SRT are completely identical. For cases where the condition number is larger, this numerical equivalence may be lost, as is demonstrated by the following least-squares problem:

$$
\underset{x \ln }{x}\left[\begin{array}{lll}
1 & 1 & 1 \\
\varepsilon & 0 & 0 \\
0 & \varepsilon & 0 \\
0 & 0 & \varepsilon
\end{array}\right] x-\left.\left[\begin{array}{l}
3 \\
\varepsilon \\
\varepsilon \\
\varepsilon
\end{array}\right]\right|_{2}
$$

where $\varepsilon$ is a very small number. The normal equations are

$$
\left[\begin{array}{ccc}
1+\varepsilon^{2} & 1 & 1 \\
& 1+\varepsilon^{2} & 1 \\
1 & 1 & 1+\varepsilon^{2}
\end{array}\right] x=\left[\begin{array}{l}
3+\varepsilon^{2} \\
3+\varepsilon^{2} \\
3+\varepsilon^{2}
\end{array}\right]
$$

When $c$ is taken, for example, equal to $10^{-8}$, With a machine preciston of $10^{-14}$, the matrix $A^{T_{A}}$ in Eq. (19) becomes singular and clearly destroys the accuracy of the $F$-test values. With the use of the MRQR algorithm and the same machine precision of $10^{-14}$, this does not occur.

\section{Conclusion}

A new technique to solve subset regression problems has been presented. The technique is called the minimal residual QR algorithm (MRQR). Basically, it performs a OR factorization with column plvoting. It has been shown analytically 
that the MRQR algorlthm can be used as a numerically stable implementation of existing stepwise regression techniques. The numerical stability is demonstrated in an experimental evaluation study.

From this technique, a reliable solution of subset regression problems has been derived that allows the use of statistical parameters commonly used In classical solutions, such as the stepwise regression technique. This new technique should allow more "accurate" models to be constructed, especially when a high correlation exists between candidate solutions in the model.

\section{References}

'Gerlach, O. H., "Analysis of a Method for Determination of Performance and Stability Derivatives in Non-stationary, Symmetric Flight," Delft Univ. of Technology, VTH-Report No. 117, 1964.

${ }^{2}$ Verhaegen, M. H., "A New Class of Algorithms in Linear System Theory: With Application to Real-Time Aircraft Model Identification," Ph.D. dissertation, Catholle Univ. Leuven, Belgium, Nov. 1985.

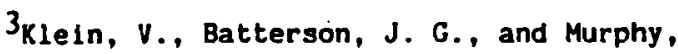
P. C., "Determination of Alrplane Structure from Flight Data by Using Modified Stepwise RegressLon," NASA TP-1916, 1981.
${ }^{4}$ Draper, N. R. and Smith, H., Applied Regression Analysis, John Wiley, NY, 1981.

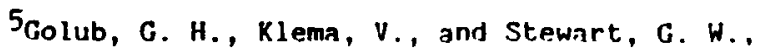
"Rank Degeneracy and Least Squares Problems,"

Technical Report TR-456, Department of Computer Science, Univ. of Maryland, College Park, 1976.

${ }^{6}$ Lawson, C. L. and Hanson, R. J., Solving Least Squares Problems, Prentice-Hall, Englewood Cliffs, NJ, 1974.

7Verhaegen, M. H., "The Minimal Residual OR Decomposition for Rellably Solving Rank Deficient Least Squares Problems," to be presented at the 1987 International Symposium on the Mathematical Theory of Networks and Systems, Phoenix, AZ, June 1987.

Bendal1, M. G. and Stuart, A., The Advanced Theory of Statistics, Vol. II, Charles Griffin and Company, Ltd., London, 1976.

9lald, A., Statistical Theory with Engineering Applications, Wiley, NY, 1952.

${ }^{10}$ Dongarra, J. J., Moler, C. B., Bunch, J. R., and Stewart, G. W. "LINPACK Users' Guide,"

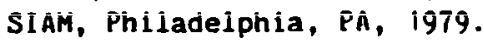

11"Matrix Users' Guide," Integrated Systems, Inc., Version 5.0, Oct. 1985.

Table 1 Data taken from Ref. 9 (p. 647) in example 1.

\begin{tabular}{|r|c|r|r|r|}
\hline \multicolumn{1}{|c|}{$x_{1}$} & $x_{2}$ & \multicolumn{1}{c|}{$x_{3}$} & \multicolumn{1}{c|}{$x_{4}$} & \multicolumn{1}{c|}{ RIIS } \\
\hline 7.0 & 26.0 & 6.0 & 60.0 & 78.5 \\
1.0 & 29.0 & 15.0 & 52.0 & 74.3 \\
11.0 & 56.0 & 8.0 & 20.0 & 104.3 \\
11.0 & 31.0 & 8.0 & 47.0 & 87.6 \\
7.0 & 52.0 & 6.0 & 33.0 & 95.0 \\
11.0 & 55.0 & 9.0 & 22.0 & 109.2 \\
3.0 & 71.0 & 17.0 & 6.0 & 102.7 \\
1.0 & 31.0 & 22.0 & 44.0 & 72.5 \\
2.0 & 54.0 & 18.0 & 22.0 & 93.1 \\
21.0 & 17.0 & 4.0 & 26.0 & 115.9 \\
1.0 & 10.0 & 23.0 & 34.0 & 83.8 \\
11.0 & 66.0 & 9.0 & 12.0 & 113.3 \\
10.0 & 68.0 & 8.0 & 12.0 & 109.1 \\
\hline
\end{tabular}


Table 2 (Retyped) computer output of MRQR on the data of Ref. 9 (p. 647).

A. SELECTING THE FIRST COLUMN FORENTRY

\begin{tabular}{|c|c|r|r|r|}
\hline SEL. \# & CAND. SOL. & $\left\|m_{S E L . \#}^{1}\right\|$ & $F_{1}$ & \multicolumn{1}{|c|}{$F_{2}$} \\
\hline 1 & $x_{1}$ & 35.5764914 & 12.6025177 & 12.6025150 \\
2 & $x_{2}$ & 30.1051204 & 21.9606017 & 21.9606160 \\
3 & $x_{3}$ & 41.3862470 & 4.1034168 & 1.4031159 \\
4 & $x_{4}$ & 29.7298993 & 22.7985202 & 22.7985270 \\
\hline
\end{tabular}

WIICH SEL \# [ENTER O TO QUIT SELECTION]: 3

DELETING A COLUMN FROM THE FIRST 1 SELECTED

\begin{tabular}{|c|c|c|c|}
\hline SEL. \# & CAND. SOL. & $F_{1}$ & $F_{2}$ \\
\hline 1 & $x_{4}$ & 22.7985202 & 22.7985270 \\
\hline
\end{tabular}

WHICH SEL, \# [ENTER O TO SKIP]: 0

B. SELECTING TIE SECOND COLUMN FOR ENTRY

\begin{tabular}{|c|r|r|r|r|}
\hline SEL. \# & CAND. SOL. & $\| m_{\text {SEL.\# }}^{2}$ & \multicolumn{1}{c|}{$F_{1}$} & \multicolumn{1}{c|}{$F_{2}$} \\
\hline 1 & $x_{1}$ & 8.6165085 & 108.2239115 & 108.2238900 \\
2 & $x_{2}$ & 29.1767726 & .1721839 & .1721817 \\
3 & $x_{3}$ & 13.2566210 & 10.2915810 & 10.2915130 \\
\hline
\end{tabular}

WHICII SEL \# [ENTER O TO QUIT SELECTION\}: 1

DELETING $\Lambda$ COLUMN FROM THE FIRST 2 SELECTED

\begin{tabular}{|c|c|c|c|}
\hline SEL. \# & CAND. SOL. & $F_{1}$ & $F_{2}$ \\
\hline 1 & $x_{4}$ & 159.2952101 & 159.2952400 \\
2 & $x_{1}$ & 108.2239115 & 108.2238900 \\
\hline
\end{tabular}

WHICI SEL \# [ENTER O TO SKIP]: 0
C. SELECTING THE THIRD COLUMN FOR ENTRY

\begin{tabular}{|c|c|r|c|c|}
\hline SEL. \# & CAND. SOL. & $\left\|m_{S E L . \#}^{3}\right\|$ & $F_{1}$ & $F_{2}$ \\
\hline 1 & $x_{2}$ & 6.9262346 & 5.0258650 & 5.0258974 \\
2 & $x_{3}$ & 7.1299118 & 4.2358160 & 1.2358519 \\
\hline
\end{tabular}

WHICH SEL \# [ENTFR O TO QUIT SELECTION]: 1

DELETING $\Lambda$ COLUMN FROM TIE FIRST 3 SELECTED

\begin{tabular}{|c|c|r|r|}
\hline SEL. \# & CAND. SOL. & \multicolumn{1}{|c|}{$F_{1}$} & \multicolumn{1}{|c|}{$F_{2}$} \\
\hline 1 & $x_{1}$ & 1.8632624 & 1.8632548 \\
2 & $x_{1}$ & 154.0076353 & 151.0080100 \\
3 & $x_{2}$ & 5.0258616 & 5.0258974 \\
\hline
\end{tabular}

WHICH SEL \# [ENTER O TO SKIP|: 0

\section{SELECXING TIELEURTILCOIUMN FOR FNTRY.}

\begin{tabular}{|c|c|r|c|c|}
\hline SEL. \# & CAND. SOL. & $\left\|m_{S E L . \# \#}^{1}\right\|$ & $F_{1}$ & $F_{2}$ \\
\hline 1 & $x_{3}$ & 6.9183549 & .0182335 & .0182345 \\
\hline
\end{tabular}

WHICH SEL \# [ENTER O TO QUIT SELECTION]: O

\section{SUMMARY}

RANK ESTIMATE: 3

PIVOT INFORMATION:

ORIGINAL COLUMN 4 PIVOTED TO COLUMN 1 ORIGINAL COIUMN 1 PIVOTED TO COIUMN 2 ORIGINAL COLUMN 2 PIVOTED TO COLUMN 3 ORIGINAL COLUMN 3 DELETED 


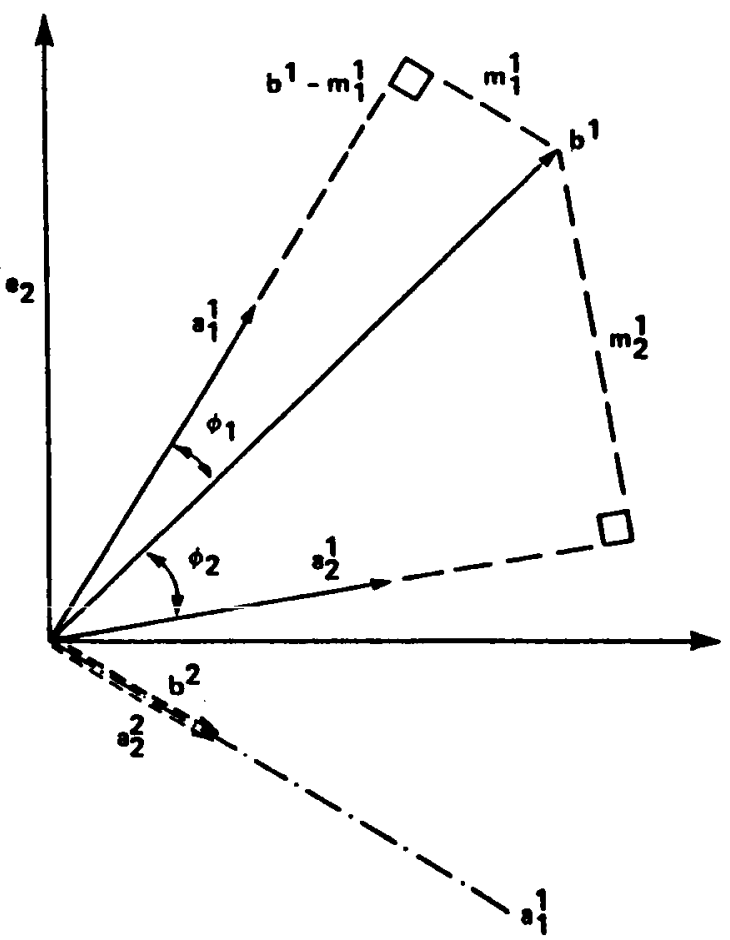

Fig. 1 The MROR algorithm for the two-dimensional case $(m=2, n=2)$.
CANDIDATE SOLUTIONS
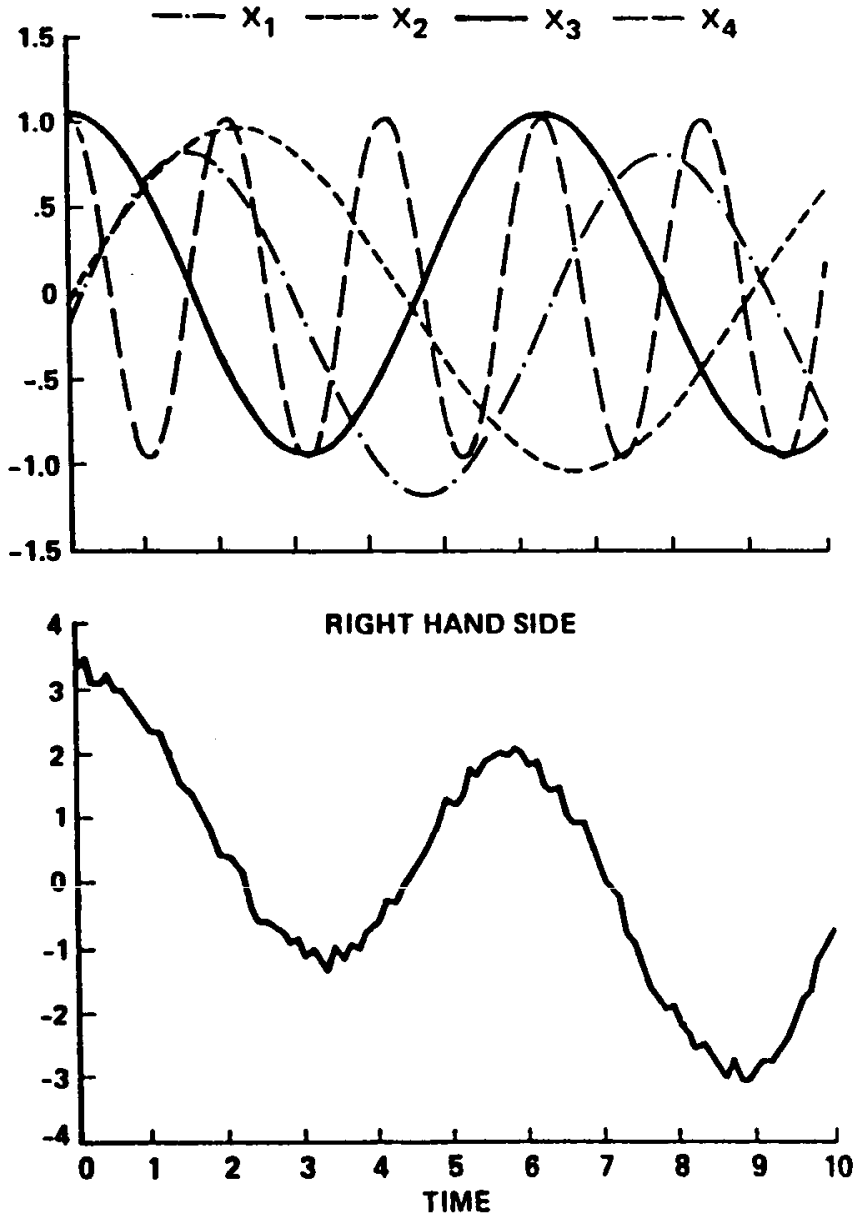

Fig. 2 Time-history plots of the candidate solutions and right-hand side before first selection In the MROR. 

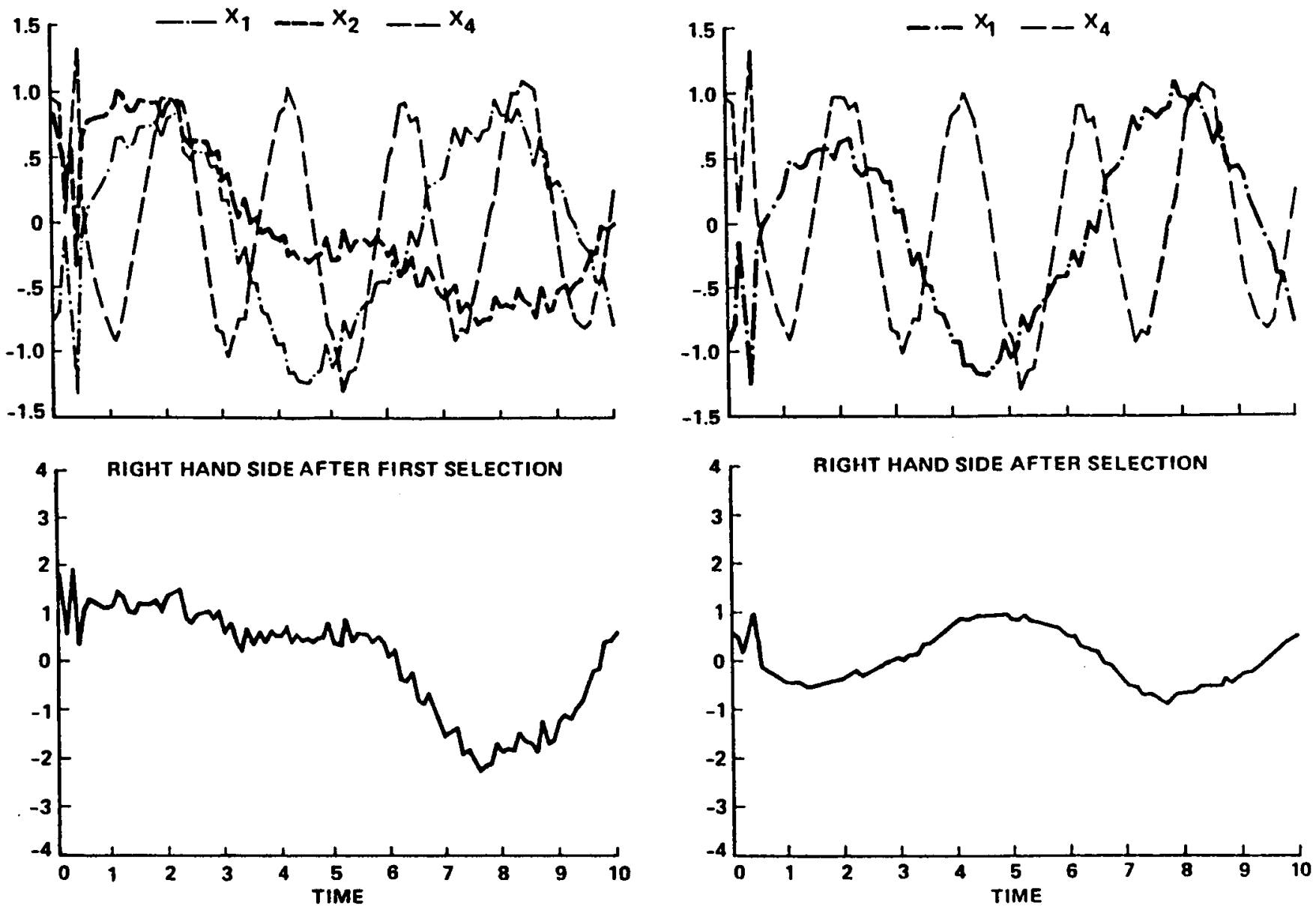

Fig. 3 Time-history plots of the candldate solutions and right-hand side after first selection ir the MRQR.

Flg. " Time-history plots of the candidate solutions and right-hand side after second selection in the MROR.

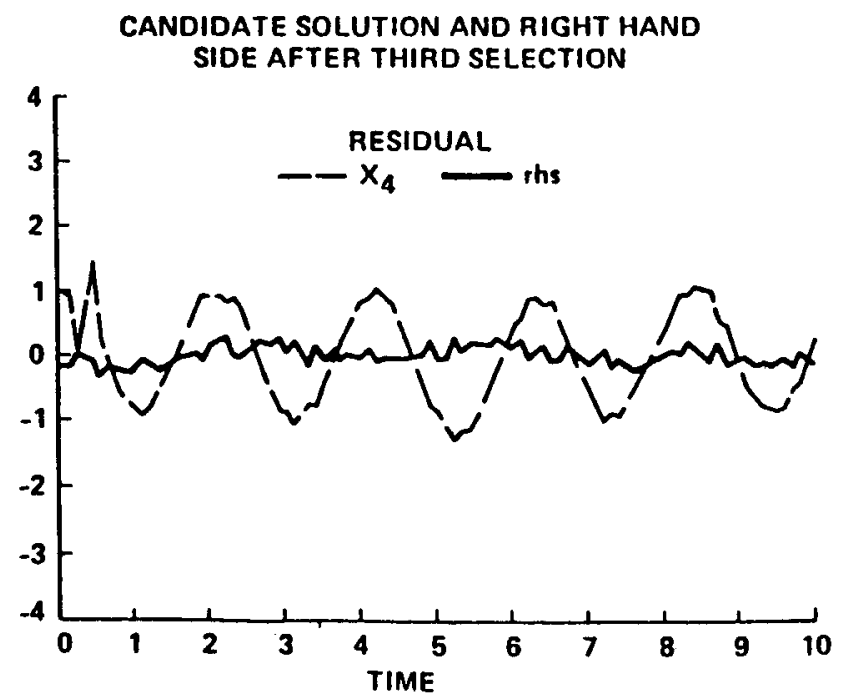

Fig. 5 Time-history plots of the candidate solutions and right-hand side after third selection in the MRQR. 


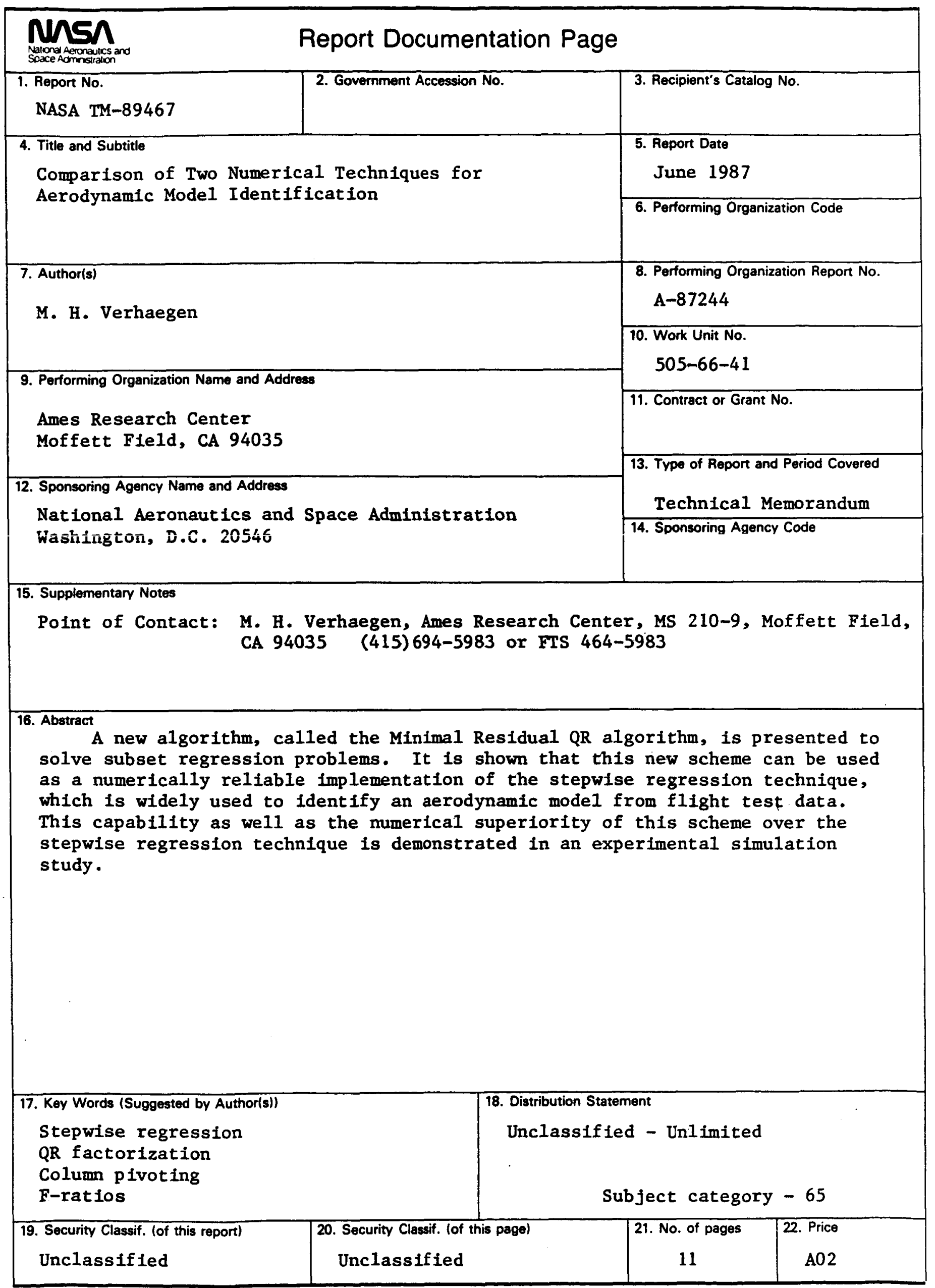

